\title{
Effects of Submerged Vegetation Density on Turbulent Flow Characteristics in an Open Channel
}

\author{
Hanqing Zhao ${ }^{1,2}$, Jing Yan ${ }^{1,2, *}$, Saiyu Yuan ${ }^{1,2} \mathbb{D}$, Jiefu Liu ${ }^{1,2}$ and Jinyu Zheng ${ }^{3}$ \\ 1 State Key Laboratory of Hydrology-Water Resources and Hydraulic Engineering, Hohai University, \\ Nanjing 210098, China; zhaohq9@126.com (H.Z.); yuansaiyu@hhu.edu.cn (S.Y.); liujiefu95@163.com (J.L.) \\ 2 College of Water Conservancy and Hydropower Engineering, Hohai University, Nanjing 210098, China \\ 3 Henan Water Conservancy Survey, Ltd., Zhengzhou 450008, China; jinyu950229@163.com \\ * Correspondence: yanjing@hhu.edu.cn
}

Received: 4 August 2019; Accepted: 12 October 2019; Published: 16 October 2019

\begin{abstract}
The vegetation density $\lambda$ affects turbulent flow type in the submerged vegetated river. This laboratory study investigates different types of vegetated turbulent flow, especially the flow at $0.04<\lambda<0.1$ and $\lambda=1.44$ by setting the experimental $\lambda$ within a large range. Vertical distributions of turbulent statistics (velocity, shear stress and skewness coefficients), turbulence kinetic generation rate and turbulence spectra in different $\lambda$ conditions have been presented and compared. Results indicate that for flow at $0.04<\lambda<0.1$, the profiles of turbulent statistics manifest characteristics that are similar to those of both the bed-shear flow and the free-shear flow, and the turbulence spectral curves are characterized with some slight humps within the low-frequency range. For $\lambda=1.44$, the turbulent statistics above the vegetation top demonstrate the characteristics of boundary-shear flow. The spectral curves fluctuate intensely within the low-frequency range, and the spectra of low-frequency eddies above vegetation top are significantly larger than the values below. The change of turbulent flow type induced by an increase of $\lambda$ would increase the maximum value of turbulence kinetic generation rate $G_{S}$ and change the point where $G_{S}$ is vertically maximum upwards to the vegetation top.
\end{abstract}

Keywords: open channel; submerged vegetation density; turbulent flow type; turbulent statistics; turbulence spectra

\section{Introduction}

Aquatic vegetation is ubiquitous in rivers, which affects the sediment transport, scalar dispersion, channel evolution and aquatic biodiversity by interfering with the flow field [1-4]. The investigation of vegetated flow can lead to a better understanding on fluvial process and hydro-environment prospect of vegetated rivers.

In an open channel with submerged vegetation, the turbulent flow type in the longitudinalvertical $(x-z)$ plane varies with an increase of vegetation density, from the bed-shear flow to the free-shear flow and the secondary boundary-shear flow $[5,6]$. The bed-shear flow happens because of bed drag near the flume bottom, with vegetation contributing to the bed roughness and increasing the bed friction [5]. For the medium dense vegetation, flow discontinuity caused by canopy drag would occur at the vegetation top, generating the free-shear flow in certain vertical region. Yan et al. [7] assumed that in extremely dense vegetation condition, flow was nearly static within canopy and flow characteristics above canopy were similar to the ones in bed-shear flow [6]. Flow in this extremely dense vegetation condition is named as the secondary boundary-shear flow with the "boundary" locating at canopy top [6]. 
Vegetation density $\lambda$ can be defined as the frontal vegetation area per unit bed area. Nepf [5] proposed that the bed-shear flow and free-shear flow occur at $0<\lambda<0.04$ and $0.1<\lambda<\lambda_{\mathrm{NB}}$, respectively. Here, $\lambda_{\mathrm{NB}}$ is the critical density which indicates the change from the free-shear flow to the secondary boundary-shear flow. The value of $\lambda_{\mathrm{NB}}$ has not been determined yet, but according to the comparison and summary of many investigations, $\lambda_{\mathrm{NB}}$ might lie in the range between 0.512 and 1.55 [6].

Previous studies have investigated the bed-shear flow $(0<\lambda<0.04)$ and the free-shear flow $\left(0.1<\lambda<\lambda_{\mathrm{NB}}\right)$, especially the latter one in the vegetated channel. Flow at $0.04<\lambda<0.1$ and $\lambda>\lambda_{\mathrm{NB}}$ (the secondary boundary-shear flow may appear) have been rarely analyzed. In the present investigation, flow characteristics have been measured and analyzed as $\lambda$ varies within a large range. Based on vertical distributions of turbulent statistics (velocity, shear stress, skewness coefficients), turbulence kinetic generation rate and turbulence spectra, different flow types have been verified. Furthermore, flow characteristics and velocity distribution laws at $0.04<\lambda<0.1$ and $\lambda>\lambda_{\mathrm{NB}}$ are discussed specifically.

\section{Experimental Setup and Measurement}

Laboratory experiments were conducted in a 12-m-long, 60-cm-wide $(B=60 \mathrm{~cm}), 60$-cm-deep recirculating flume (Figure 1). An ultrasonic flow meter (Krohne, Germany) with an accuracy of $0.001 \mathrm{~L} / \mathrm{s}$ was used to monitor flow discharge $Q$, which was maintained constant by a variable frequency pump system. The flow from the water tank passed through a flow-straightener upstream of the vegetation section to smooth the inflow. A tailgate was mounted at the outlet of the flume to control the flow depth $H$ in the experiment. The bed slope was adjustable to create longitudinally uniform flow, which was judged according to the flow depths measured along the streamwise direction. Flow depth was measured by rulers with an accuracy of $0.5 \mathrm{~mm}$ placed on the glass sidewall at a spacing of $1 \mathrm{~m}$.

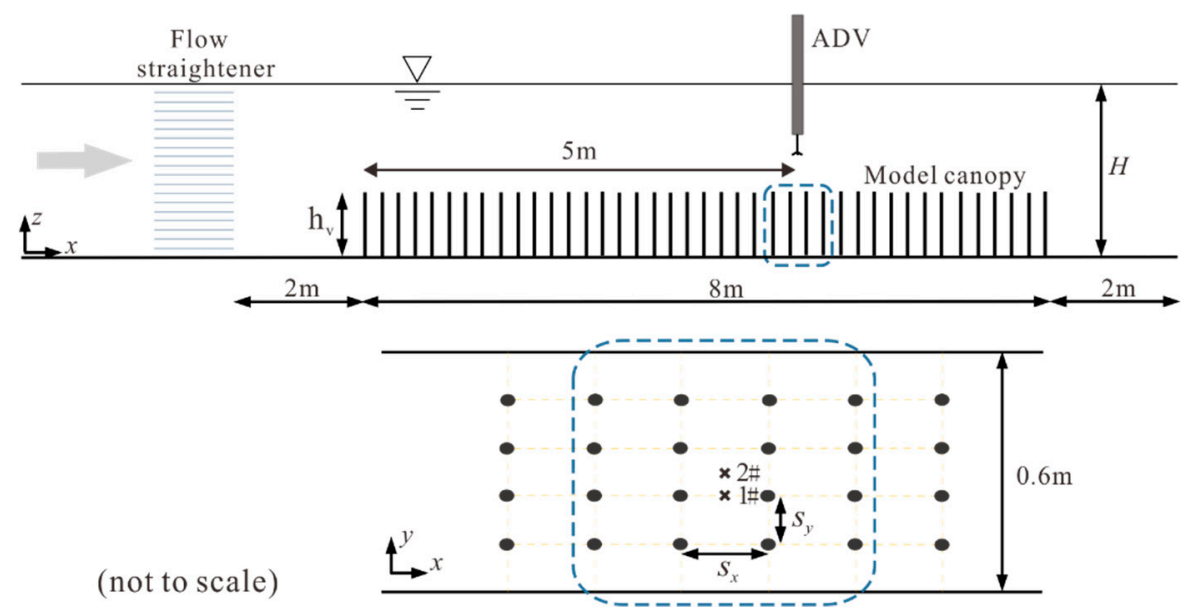

Figure 1. A schematic of the experimental setup and measurement sections.

Vegetation was simulated by $6-\mathrm{cm}$-high (i.e., $h_{\mathrm{v}}=6 \mathrm{~cm}$ ) aluminum cylinder with diameter $d_{\mathrm{v}}=0.6 \mathrm{~cm}$. In order to place the cylinders in the channel bed, an artificial bottom was constructed with an 8-m-long plate of 0.6-cm-holed pegboard. The streamwise and spanwise spacings between neighboring cylinders were defined as $S_{x}$ and $S_{y}$ respectively. The vegetation was linearly arranged at the mid $8 \mathrm{~m}$ length of the flume, and $\lambda$ was calculated by $\lambda=\left(d_{\mathrm{v}} h_{\mathrm{v}}\right) /\left(S_{x} S_{y}\right)$.

$3 \mathrm{D}$ flow velocity was measured by an acoustic Doppler velocimeters (ADV, Nortek, Norway) with an accuracy of $\pm 1 \mathrm{~mm} / \mathrm{s}$, mounted on an automatic traversing system that could move in three orthogonal directions with a step of $0.1 \mathrm{~mm}$. The sampling period was set to be 180 seconds with a frequency of $200 \mathrm{~Hz}$. The device with four probes had a 5-cm blind zone near the water surface for velocity measurement, which was, therefore, supplemented by another device with lateral probes.

Velocity profiles were measured at the section $5 \mathrm{~m}$ downstream of the leading edge of the canopy area, where the flow was fully developed in the longitudinal direction. Velocities at two locations 
(shown as locations 1\# and 2\# in Figure 1) in the test area were measured and averaged to represent the overall flow.

Flow measurements were started at $z=1 \mathrm{~mm}$ at intervals of $\Delta z=2 \mathrm{~mm}$ moving upwards to the free surface. As the stems did not allow the access of ADV probes, velocities within $2.1 \mathrm{~cm}$ above the channel bed at location 1\# could not be measured. Flow in this region is characterized with wake turbulence that changes little with the cylinders heights. Therefore, this measurement limitation was overcome by replacing two obstructive cylinders of $6 \mathrm{~cm}$ height with cylinders of $4 \mathrm{~cm}$ height.

This experimental investigation is mainly to investigate the influence of $\lambda$; therefore, we maintained the flow depth $H$ and the flow discharge $Q$ constant for different cases. Hence, the relative submergence $S u b=H / h_{\mathrm{v}}$ and the mean bulk velocity $U_{m}=Q /(\mathrm{BH})$ remain constant in the experiment. The flow with $S u b=3$ and $U_{m}=30 \mathrm{~cm} / \mathrm{s}$ in this study represented the high-speed shallow flow. The experimental $\lambda$ was selected from a wide range to create different flow conditions. According to [5], the bed-shear flow and free-shear flow may occur in Cases I \& II $(\lambda=0,0.0056)$ and Case V $(\lambda=0.36)$, respectively (Table 1).

Table 1. Experimental conditions.

\begin{tabular}{cccccccc}
\hline Case & $\lambda$ & $S_{\boldsymbol{x}}(\mathbf{c m})$ & $\boldsymbol{S}_{\boldsymbol{y}}(\mathbf{c m})$ & $\boldsymbol{H}(\mathbf{c m})$ & $\boldsymbol{S} \boldsymbol{u b}$ & $\boldsymbol{Q}(\mathrm{L} / \mathbf{s})$ & $\boldsymbol{U}_{\boldsymbol{m}}(\mathbf{c m} / \mathbf{s})$ \\
\hline I & 0 & 0 & 0 & 18 & - & 32.44 & 30.04 \\
II & 0.0056 & 40 & 16 & 18 & 3 & 32.34 & 29.94 \\
III & 0.045 & 10 & 8 & 18 & 3 & 32.43 & 30.03 \\
IV & 0.09 & 5 & 8 & 18 & 3 & 32.35 & 29.95 \\
V & 0.36 & 5 & 2 & 18 & 3 & 32.4 & 30 \\
VI & 1.44 & 2.5 & 1 & 18 & 3 & 32.48 & 30.07 \\
\hline
\end{tabular}

Velocity profiles at another 9 locations (from location a\# to location i\# in Figure 2) in flow field for Case $\mathrm{V}$ were measured additionally to study spatial variations of the flow characteristics and the representative locations of the canopy flow. The 9 locations were distributed evenly and fully within the bed area of $S_{x} \times S_{y}$ It is, thus, believed the spatial averages of the experimental data at these 9 locations can represent the overall flow. A comparison between the averaged velocity at the 9 locations and the averaged velocity at points $1 \#$ and $2 \#$ was conducted to verify that the measurements for points $1 \# \&$ 2\# are indeed representative.

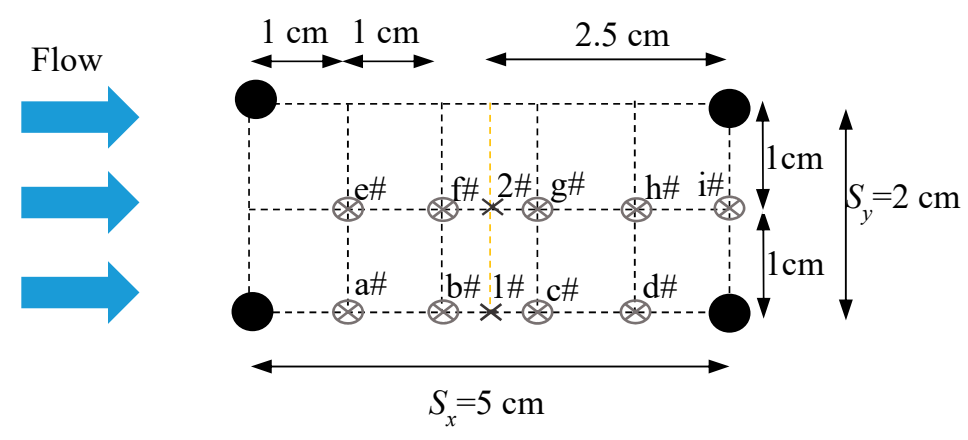

Figure 2. The arrangement of measurement points for analysis of spatial variations and representative locations of the flow field.

\section{Results}

\subsection{Spatial Variation of the Velocity and Reynolds Stress Profiles}

Figures 3 and 4 compare the velocity profiles and the Reynolds stress distributions at different locations for Case V. The values characterized with subscripts of "a\# i\#", "1\# 2\#", "a\# d\#" and "e\# i\#" denote the space-averaged values from location a\# to i\#, 1\# to $2 \#$, a\# to $\mathrm{d} \#$ and e\# to i\#, 
respectively. Therefore, $U_{\mathrm{a \#} \sim \mathrm{i} \#}$ and $-\overline{u w}_{a \# \sim \text { i\# }}$ represent the overall space-averaged flow characteristics in the experiment.
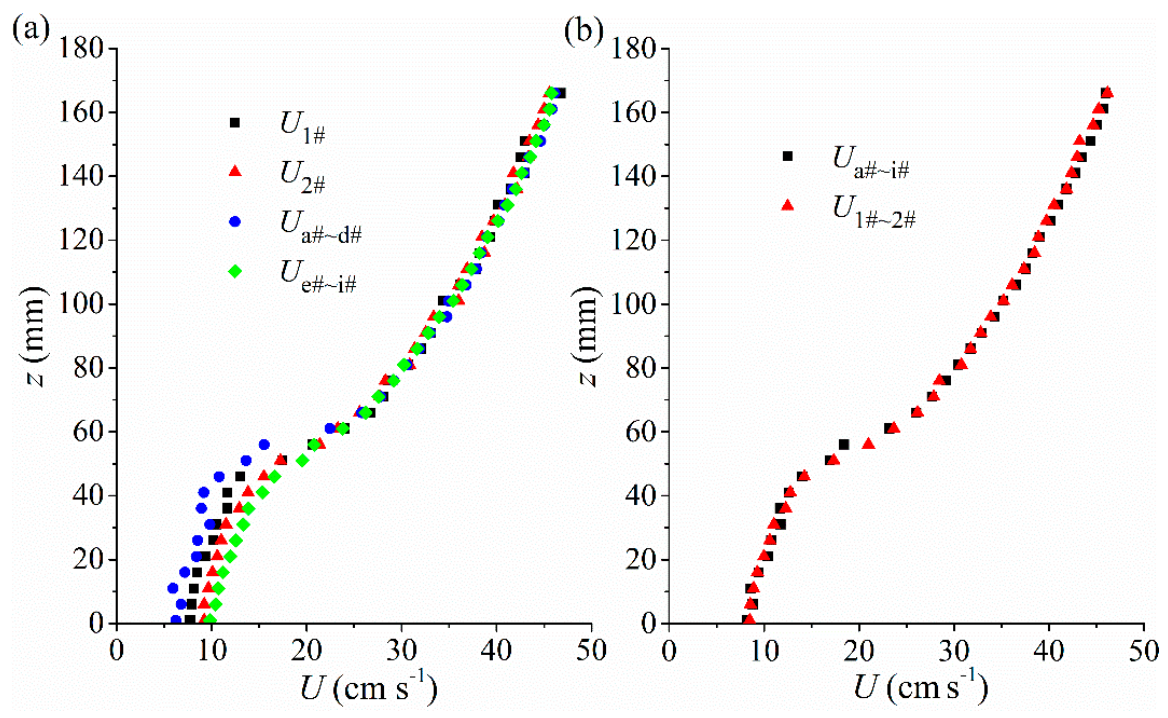

Figure 3. (a) Spatial variations of the velocity profiles and (b) the comparison between $U_{1 \# \sim 2 \# \text { profile }}$ and the $U_{\mathrm{a} \# \sim \mathrm{i} \#}$ one in Case $\mathrm{V}$.
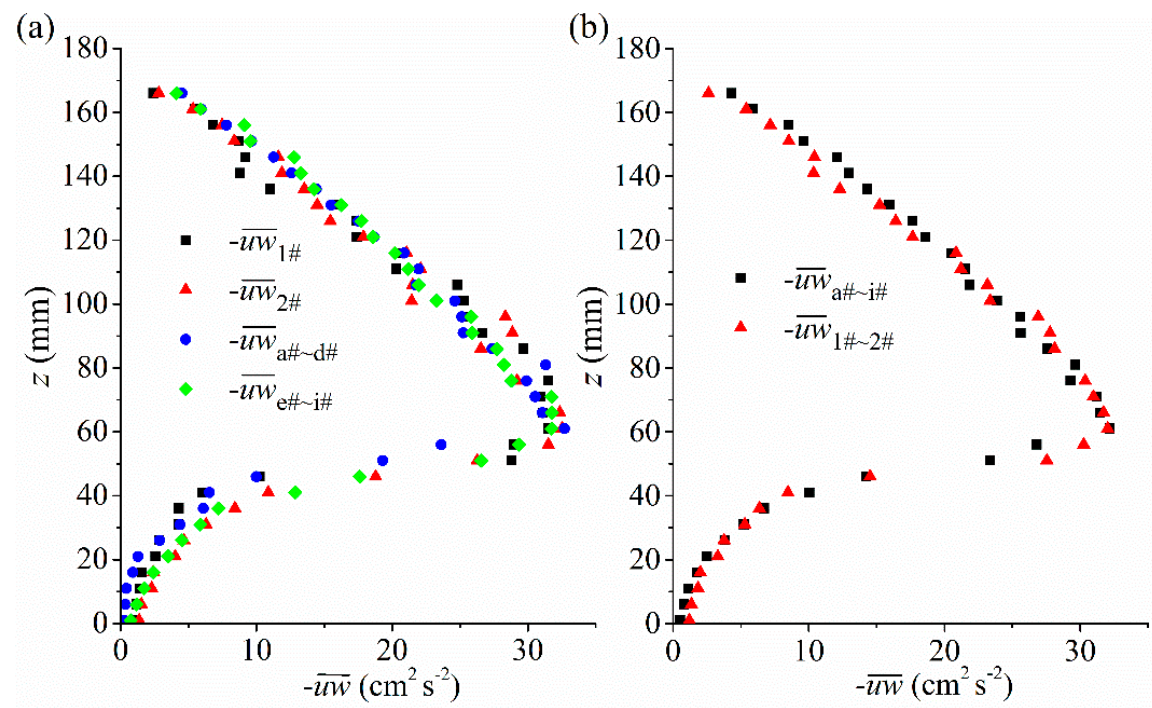

Figure 4. (a) Spatial variations of the Reynolds stress profiles and (b) the comparison between $-\overline{u w}_{1 \# \sim 2 \#}$ profile and the $-\overline{u w}_{a \# \sim i \#}$ one in Case V.

The velocity $U$ and Reynolds shear stress $-\overline{u w}(u$ and $w$ denote velocity fluctuations in the $x$ and $z$ directions, respectively) vary horizontally in canopy layer meanwhile the profiles for different locations concentrate above the canopy (Figures $3 \mathrm{a}$ and $4 \mathrm{a}$ ). The flow shear in the free stream (Locations e\# i\# \& 2\#) with larger $U$ and $-\overline{u w}$ is comparatively intensive than that behind the vegetation (Locations a\# $\mathrm{d} \# \& 1 \#)$. The results that $U_{1 \#}>U_{\mathrm{a} \# \sim \mathrm{d} \#,} U_{2 \#}<U_{\mathrm{e} \# \mathrm{i} \#}$ and $U_{1 \#}<U_{2 \#}$ are shown in Figure 3a. However, $-\overline{u w}_{1 \#} \approx-\overline{u w}_{a \# \sim d \#,}-\overline{u w}_{2 \#} \approx-\overline{u w}_{e \# \sim \text { i\# }}$ and $-\overline{u w}_{1 \#}<-\overline{u w}_{2 \#}$ are shown in Figure 4a.

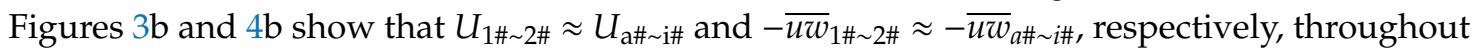
the depth. Hence, it is sufficiently accurate to describe the overall flow dynamics by the averaged velocity of points $1 \#$ and 2\#. In this study, therefore, we analyze the flow characteristics by the averaged values of points $1 \#$ and $2 \#$. The subscript is omitted in the rest sections of this article. 


\subsection{Turbulent Statistics}

\subsubsection{Velocity}

Figure 5 presents the velocity profiles for different $\lambda$, and the averaged velocity gradients $\mathrm{d} U / \mathrm{d} z$ in specific regions have been listed in Table 2. In order to show each profile clearly, the profiles are plotted in two pictures. The vertical regions where $U$ increase considerably are marked with red curves in Figure 5.

(a)

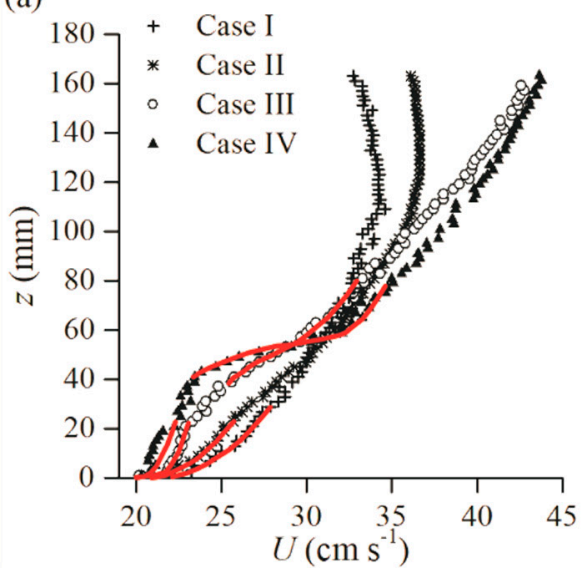

(b)

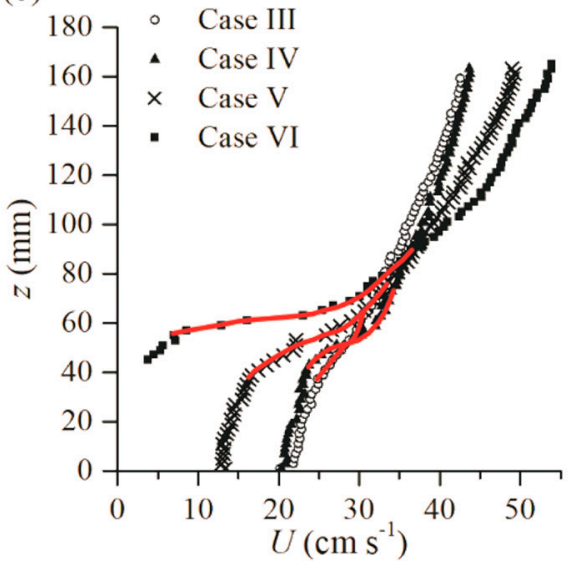

Figure 5. $U$ profiles with different vegetation densities, (a) from Case I to Case IV and (b) from Case III to Case VI.

Table 2. The velocity gradient $\mathrm{d} U / \mathrm{d} z\left(\mathrm{~s}^{-1}\right)$ in different regions.

\begin{tabular}{ccccccc}
\hline & Case I & Case II & Case III & Case IV & Case V & Case VI \\
\hline $0<z<10 \mathrm{~mm}$ & 2.99 & 3.462 & 2.407 & 2.03 & -0.068 & - \\
$55 \leq z \leq 65 \mathrm{~mm}$ & 1.126 & 1.38 & 2.025 & 3.077 & 4.978 & 19.764 \\
\hline- indicates that the value is not
\end{tabular}

The velocity $U$ increases considerably near the channel bed $(0<z<10 \mathrm{~mm})$ for Cases I \& II (Figure 5a \& Table 2), which shows the characteristics of the bed-shear flow [8], and $\mathrm{d} U / \mathrm{d} z=3 \mathrm{~s}^{-1}$ in this region. For Case V, $U$ increases rapidly near the canopy top $(55 \leq z \leq 65 \mathrm{~mm})$ and $\mathrm{d} U / \mathrm{d} z=5 \mathrm{~s}^{-1}$ there (Figure $5 b$ \& Table 2), which indicates intensive shear exists in this region under this flow condition.

For Cases III \& IV which $0.04<\lambda<0.1$, the velocity $U$ increases considerably both near the bed and at the canopy top (Figure $5 \mathrm{a} \&$ Table 2). $\mathrm{d} U / \mathrm{d} z$ vary from 2 to $2.4 \mathrm{~s}^{-1}$ near the channel bed and 2 to $3 \mathrm{~s}^{-1}$ around the vegetation top, which are between the ones for Cases I \& II (bed-shear flow) and the one for Case $\mathrm{V}$ (free-shear flow) (Table 2).

In Case VI, the velocity profile along the water depth is highly similar to the one presented in [9] with $\lambda=1.544$ and increases sharply above the canopy but is small within the canopy (Figure $5 \mathrm{~b}$ ). $\mathrm{d} U / \mathrm{d} z$ around the canopy top is far greater than those of other cases. That indicates intensive stress appears at the canopy top, and the canopy top can be considered as a "new bed" at this density.

\subsubsection{Shear Stress}

The Reynolds shear stress $-\overline{u w}$ increases along $z$ in a certain region near the bed and then decreases towards the free surface for all the cases (Figure 6). For Cases I \& II (bed-shear flow), the shear stress $-\overline{u w}$ attains its peak at $z=(0.15 \sim 0.2) H$ (Figure $6 \mathrm{a})$, which is the upper boundary of the inner region [7]. The height of the $-\overline{u w}$ peak for Case II (with vegetation) is higher than the one for Case I (with no vegetation) (Figure 6a). In Case V, the peak of $-\overline{u w}$ appears at the canopy level $z=h_{\mathrm{V}}$ (Figure 6b) and the free-shear flow happens because of flow discontinuity at the canopy top [10]. 
(a)

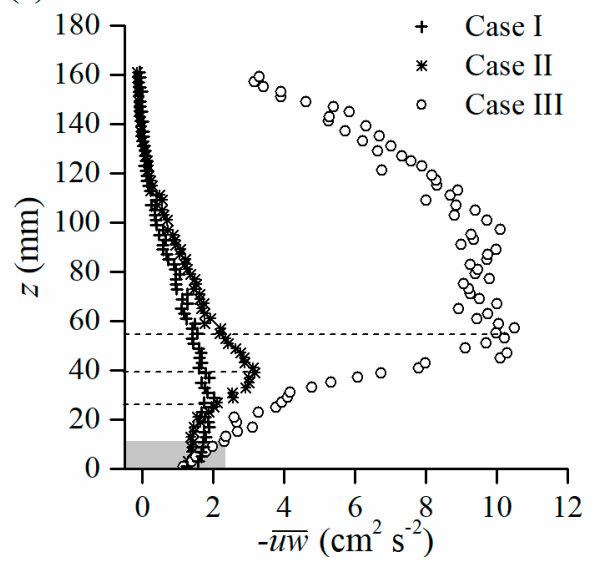

(b)

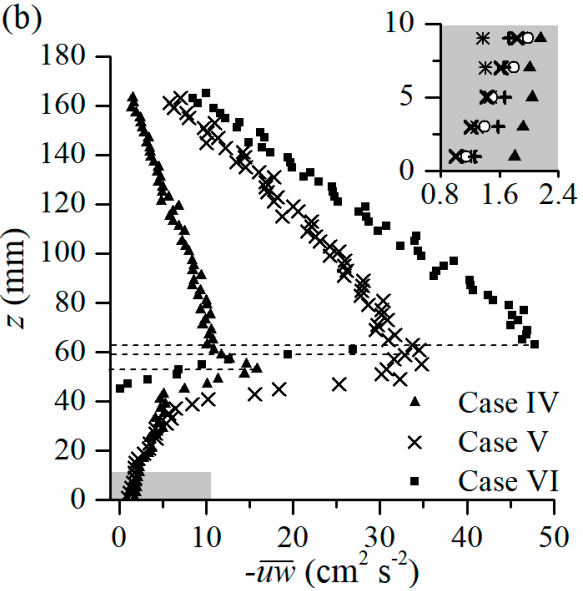

Figure 6. $-\overline{u w}$ profiles with different vegetation densities, (a) from Case I to Case III and (b) from Case IV to Case VI.

The $-\overline{u w}$ profile in Case VI is similar to the one in Case V, except that the $-\overline{u w}$ is nearly zero within canopy. In Cases III \& IV, the maximum $-\overline{u w}$ appears between the one for Cases I \& II (bed-shear flow) and the one for Case V (free-shear flow) in Figure 6.

In the region near the bed, the largest $-\overline{u w}$ appears in Case IV $(\lambda=0.09)$ comparing with other cases (see the partially enlarged figure in Figure 6b). Although the difference is small among the 6 cases, the result could yet be applied to explain why the flow velocity for sediment incipient motion is minimum or the bed load transport rate is maximum while $\lambda$ varies around 0.09 [11,12].

\subsubsection{Skewness Coefficients}

Turbulence affects bursting events along the depth, and the bursting phenomenon could be judged according to the skewness coefficients [9]. The skewness coefficients are defined as:

$$
\begin{aligned}
& S k_{u}=\frac{1}{N} \sum_{i=1}^{N} u^{3} /\left(\sqrt{\frac{1}{N} \sum_{i=1}^{N} u^{2}}\right)^{3} \\
& S k_{w}=\frac{1}{N} \sum_{i=1}^{N} w^{3} /\left(\sqrt{\frac{1}{N} \sum_{i=1}^{N} w^{2}}\right)^{3}
\end{aligned}
$$

where $N$ is the sampling number, $S k_{u}$ and $S k_{w}$ are the skewness coefficients of $u$ and $w$, respectively. Bursting phenomenon manifests in four different manners, i.e., outward interaction $\left(S k_{u}>0, S k_{w}>0\right)$, ejection $\left(S k_{u}<0, S k_{w}>0\right)$, inward interaction $\left(S k_{u}<0, S k_{w}<0\right)$ and sweep $\left(S k_{u}>0, S k_{w}<0\right)$ [8]. The absolute values of $S k_{u}$ and $S k_{w}$ reflect the intensity degree of the bursting actions [13].

Sweep occurs in the lower flow zone while ejection appears in the upper zone for all the experiment cases (Figure 7).

For Cases I \& II (Figure 7a,b), the sweep is the most intensive at the flume bed. The ejection achieves the utmost at the mid flow depth ( $z=93 \mathrm{~mm}$ in Case I and $105 \mathrm{~mm}$ in Case II), which is caused by the sidewall effect in a narrow channel [13]. The strongest sweep appears at $z=45 \mathrm{~mm}$, while the ejection is the fiercest at the water surface for Case V (Figure 7e).

When it comes to Case VI, the $S k_{u}$ and $S k_{w}$ profiles above canopy (Figure 7f) are similar to those throughout depth in the bed-shear flow. Meanwhile, the ejection action is vertically fiercest near the free surface (Figure 7f), indicating that the sidewall effect disappears in Case VI. 
The bursting events for Cases III \& IV manifest the characteristics of both the free-shear flow and bed-shear flow (Figure $7 c, d): S k_{w}$ profiles are similar to the one for the free-shear flow; however, $S k_{u}$ attains its vertical maximum at the bottom, which is in line with that for the bed-shear flow.
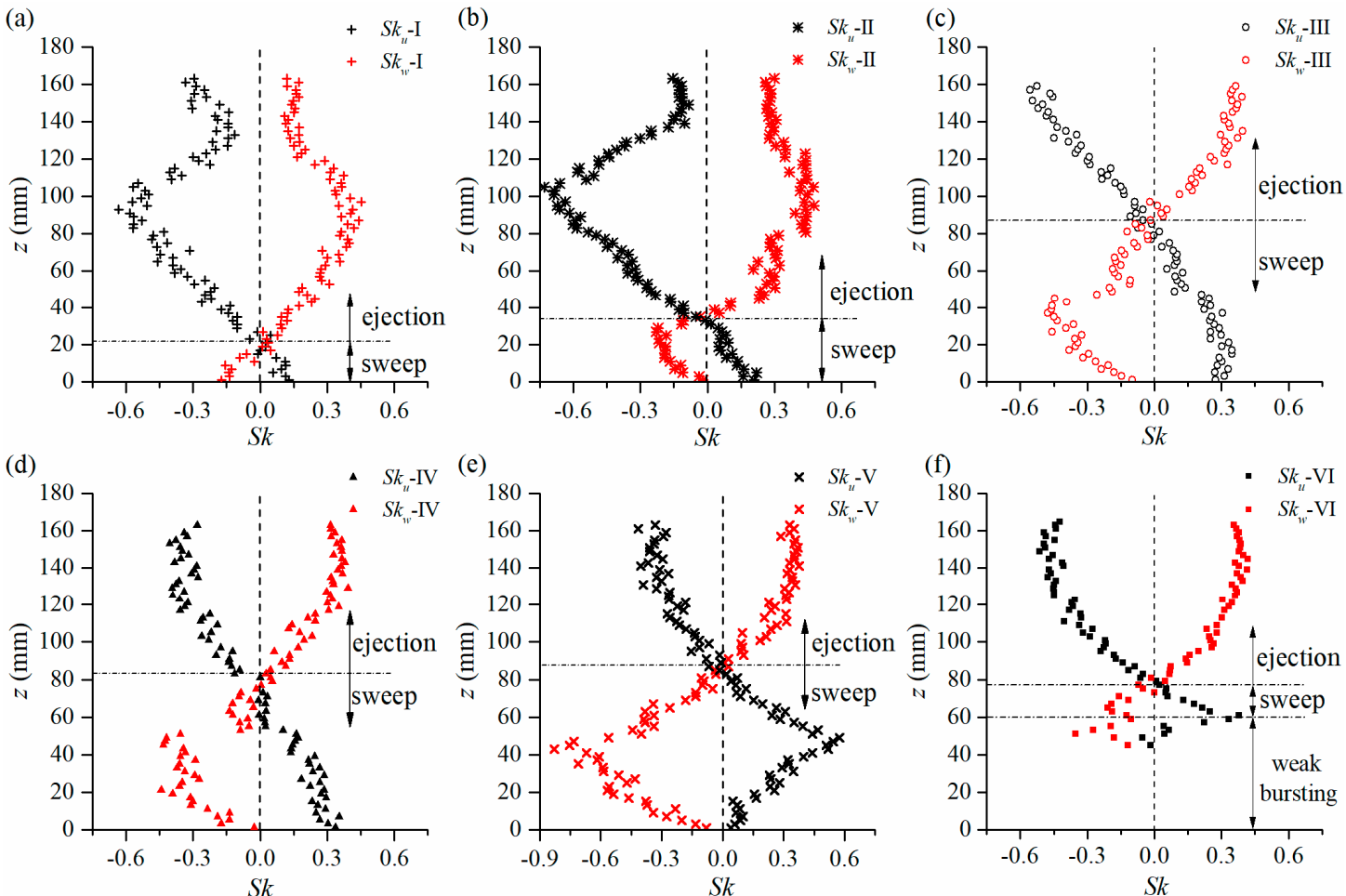

Figure 7. $S k_{u}$ and $S k_{w}$ with different vegetation densities, in (a) Case I, (b) Case II, (c) Case III, (d) Case IV, (e) Case V and (f) Case VI.

\subsection{Turbulence Kinetic Energy Generation Rate}

The turbulence kinetic energy generation rate $G_{S}, G_{S}=-\overline{u w} \frac{\partial U}{\partial z}$, represents the turbulent energy that is drained from the mean flow. We compute $G_{S}$ by the measured data and plot $G_{S}$ profiles corresponding to different vegetation densities in Figure 8.
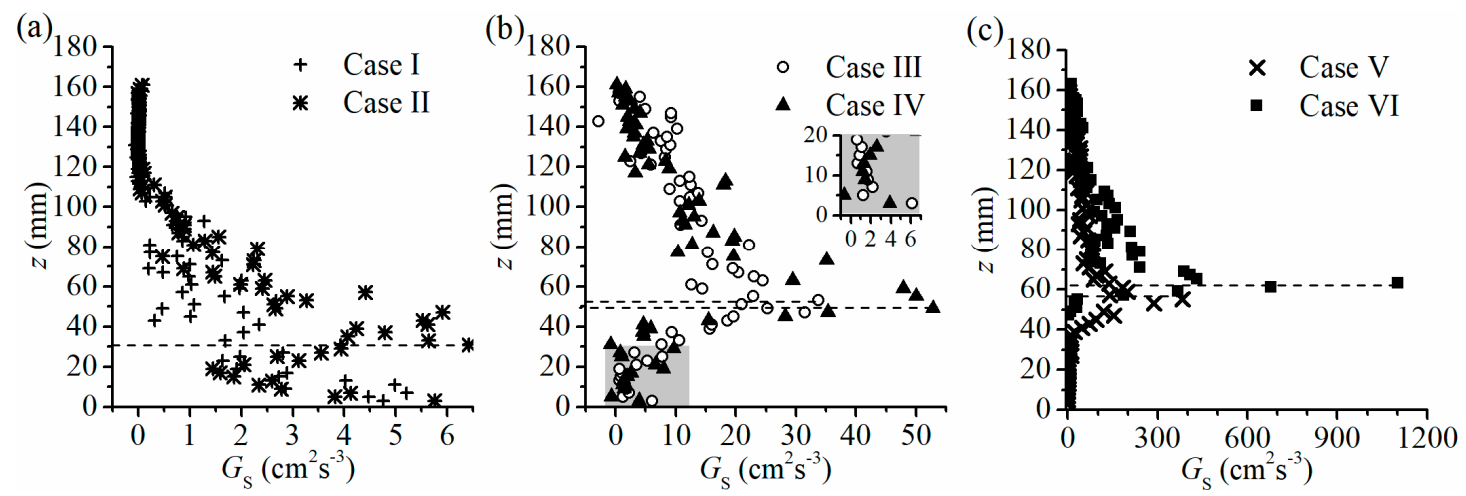

Figure 8. GS profiles with different vegetation densities, in (a) Cases I \& II, (b) Cases III \& IV and (c) Cases V \& VI.

For Case I (bed-shear flow with no vegetation), $G_{S}$ decreases with $z$ and its vertical maximum appears at the channel bottom. However, for Case II (the bed-shear flow with sparse vegetation), $G_{S}$ attains its peak both at the flume bottom and at a certain height above the bed $(z=31 \mathrm{~mm})$. Meanwhile, $G_{S}$ in Case II is larger than that in Case I. 
In Cases III \& IV, the height of vertical maximum $G_{S}$ is slightly below the vegetation top (Figure $8 b$ ). $G_{S}$ within canopy decreases towards the bed but increases suddenly on the bed (see the partially enlarged figure in Figure $8 b$ ). The near bed $G_{S}$ values approximate the ones in Cases I \& II.

The maximum of $G_{S}$ is increasing continuously from Cases I \& II, Case III \& IV, Case V to Case VI (Figure 8), indicating that the change of the turbulent flow type, which is induced by an increase of $\lambda$, would intensify the flow turbulence.

\subsection{Turbulence Spectra}

The turbulence spectra $F(f)$ represents the turbulence kinetic energy of eddies with different frequencies $f$. Basing on the Taylor's frozen hypothesis, the $F(f) \sim f$ relation could be obtained by the Fourier Transformation of the velocity series at any point. The maximum time lag was set at $23 \%$ of the time series length in this study.

Figure 9 presents the spectra of longitudinal velocities along the vertical direction. For turbulence around the vegetation top the spectral densities increase with $\lambda$ in Figure 9, which coincides with the influence of $\lambda$ on the maximum $G_{\mathrm{S}}$ among different cases (Figure 8). The intermediate frequency in this study except $z=51 \mathrm{~mm}$ in Case VI ranges from 0.4 to $4 \mathrm{~Hz}$ where the $-5 / 3$ power law is satisfied, which is characteristic of the systematic cascade of turbulence energy within the inertial subrange (ISR).
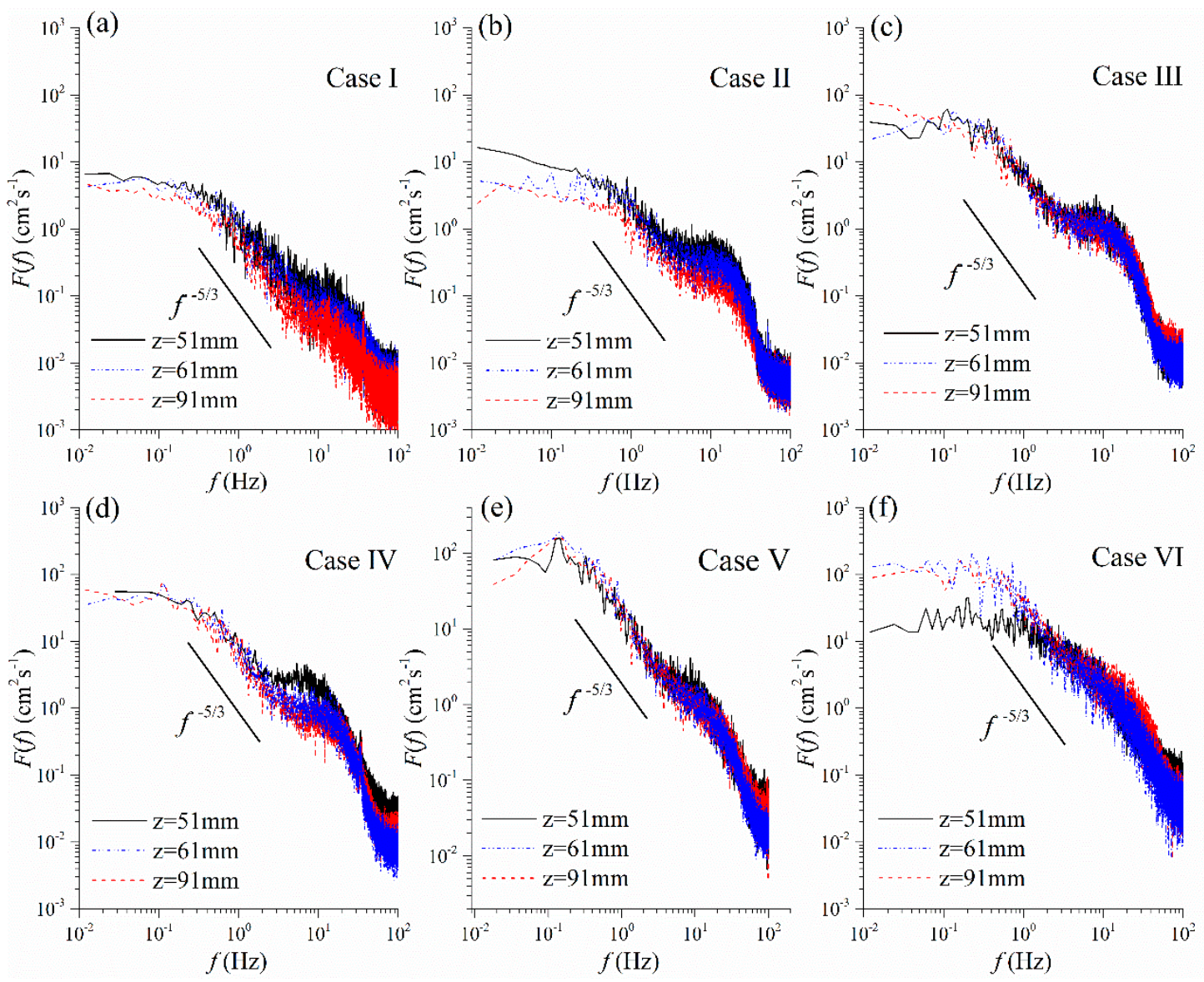

(e)
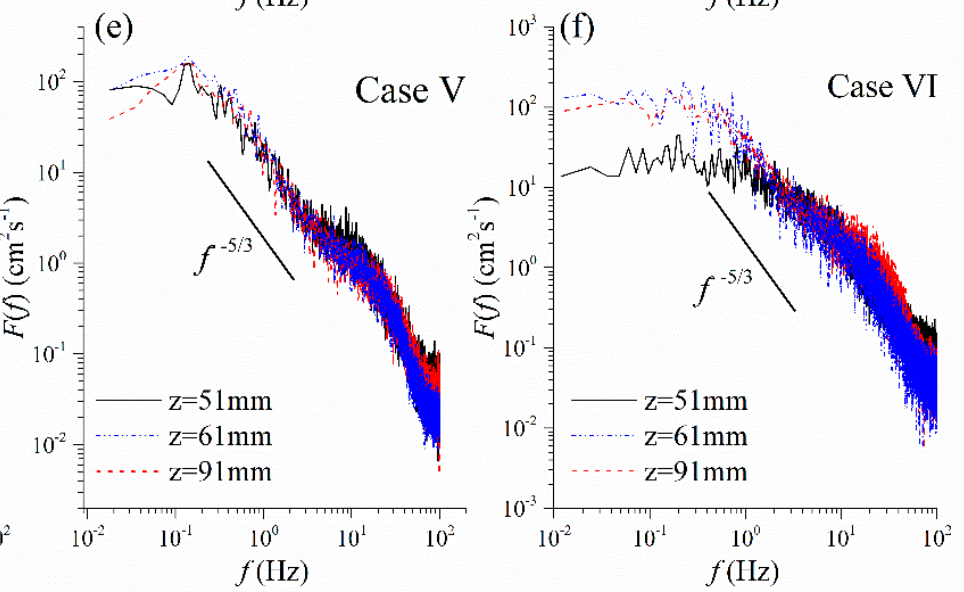

Figure 9. Turbulence spectra $F(f)$ at different heights in (a) Case I, (b) Case II, (c) Case III, (d) Case IV, (e) Case V and (f) Case VI.

In the low-frequency range below ISR, the spectral curves vary along the vertical, and the change tendency is affected by the turbulent flow type. For the bed-shear flow in Cases I \& II, especially the one at $z=51$ or $91 \mathrm{~mm}, F(f)$ tends to decrease slightly with $f$, which coincides with the one presented in an open channel with no vegetation [14]. The low frequency eddies would evolve upwards towards 
the free surface, so that the corresponding $F(f)$ decreases from $z=51 \mathrm{~mm}$ to $z=61$ and $91 \mathrm{~mm}$ in Figure 9a,b. For the free-shear flow in Case V, the spectral curve peaks at $0.15 \mathrm{~Hz}$ and the peak frequency does not change with vertical height (Figure 9e), as mentioned by [15] in a study of the free-shear flow. The spectral curve is the highest at $z=61 \mathrm{~mm}$ (i.e., the vegetation top) because the flow shears intensely there.

For Cases III \& IV at $0.04<\lambda<0.1$, there are some slight spectral humps whose frequencies vary around $0.15 \mathrm{~Hz}$. The peak spectra in these conditions are smaller than the one in the free-shear flow because of the relatively weak turbulence caused by discontinuity in flow resistance. The $F(f)$ changes little with the gauging height in Figure 9c,d.

For Case VI, the spectral curve fluctuates intensely within the low-frequency range. The curve at $z=61 \mathrm{~mm}$ nearly coincides with the one at $z=91 \mathrm{~mm}$ and is higher than the one at $z=51 \mathrm{~mm}$. The height of $z=51 \mathrm{~mm}$ locates within the canopy, where the low speed porous media flow might happen in this dense vegetation condition with $\lambda=1.44$.

\subsection{Distribution Law of the Velocity Profiles}

For bed-shear turbulent flow, the $U$ profile is logarithmic in a certain vertical region (i.e., the inertial layer) [16]. We define the lower boundary height of the inertial layer as $h_{\mathrm{c} 1}$ where the $U$ profile deviates from the logarithmic law toward the bed. Figure 10a shows that the inertial layer starts from the channel bed (i.e., $h_{\mathrm{c} 1}=0$ ), when there is no-vegetation (Case I, the red line); while it locates above the bed (i.e., $h_{\mathrm{c} 1}>0$ ) in case of a sparsely vegetated condition (Case II, the black line). For the roughness layer $\left(0<z<h_{\mathrm{c} 1}\right)$ below in Case II, the exponential function [17] has been adopted to fit the measured $U$ data, which leads to the coefficients of determination $R^{2}=0.9229$.
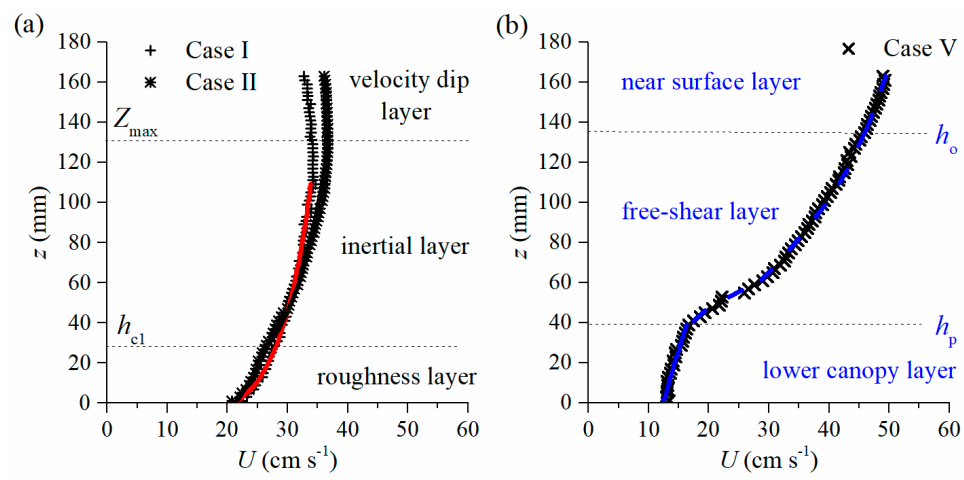

Figure 10. $U$ Profiles along the depth for (a) Cases I \& II and (b) Case V.

In Figure 10a, maximum $U$ appears below the free surface because of the sidewall effect in the cross-section, which means velocity dip phenomenon. This phenomenon had been observed in a narrow open channel flow [14] and in the vegetated flow [18]. We define the height of the maximum velocity as $Z_{\max }$ and the region from $z=Z_{\max }$ to the free surface as the velocity dip layer, respectively. The $U$ profile in the velocity dip layer could be described by a power law, with $R^{2}=0.9015$ for Case II (Figure 10a).

Case $\mathrm{V}$ is characterized with the free-shear flow, which could be divided into three layers as the lower canopy layer $\left(0<z<h_{\mathrm{p}}\right)$, the intermediate free-shear layer $\left(h_{\mathrm{p}}<z<h_{\mathrm{o}}\right)$ and the surface layer $\left(h_{\mathrm{o}}<z<H\right.$ ) [9]. $h_{\mathrm{p}}$ and $h_{\mathrm{o}}$ are the boundaries of the free-shear layer that could be estimated by the displacement height and the turbulent kinetic energy balance, respectively $[19,20]$. Experimental $U$ data in the free-shear layer and the surface layer were fitted by the hyperbolic tangent function with $R^{2}=0.9986$ and logarithmic function with $R^{2}=0.986$ (see the blue dash line in Figure 10b), respectively. The $U$ data in the lower canopy layer was compared with the power, exponential and logarithmic functions, of previous studies $[17,18,21]$, separately. An exponential distribution law is satisfied in this region with $R^{2}=0.9328$ (see Figure 10b). 
For flow at $0.04<\lambda<0.1$, i.e., Cases III \& IV in this study, the measured $U$ profiles were mathematically fitted according to characteristics of the free-shear flow and bed-shear flow, separately. With consideration of the free-shear flow features, the coefficients of determination $R^{2}=0.9987$ (Case III) and 0.9985 (Case IV) (hyperbolic tangent distribution law in the free-shear layer), and $R^{2}=0.8372$ (Case III) and 0.8993 (Case IV) (exponential law in the lower canopy layer) (see the blue lines in Figure $11 \mathrm{a}, \mathrm{b})$. We find that $U$ profiles throughout the region above canopy could be described by the hyperbolic tangent law. Therefore, one could assume that the free-shear layer is unconfined and the surface layer "disappears" in these cases.
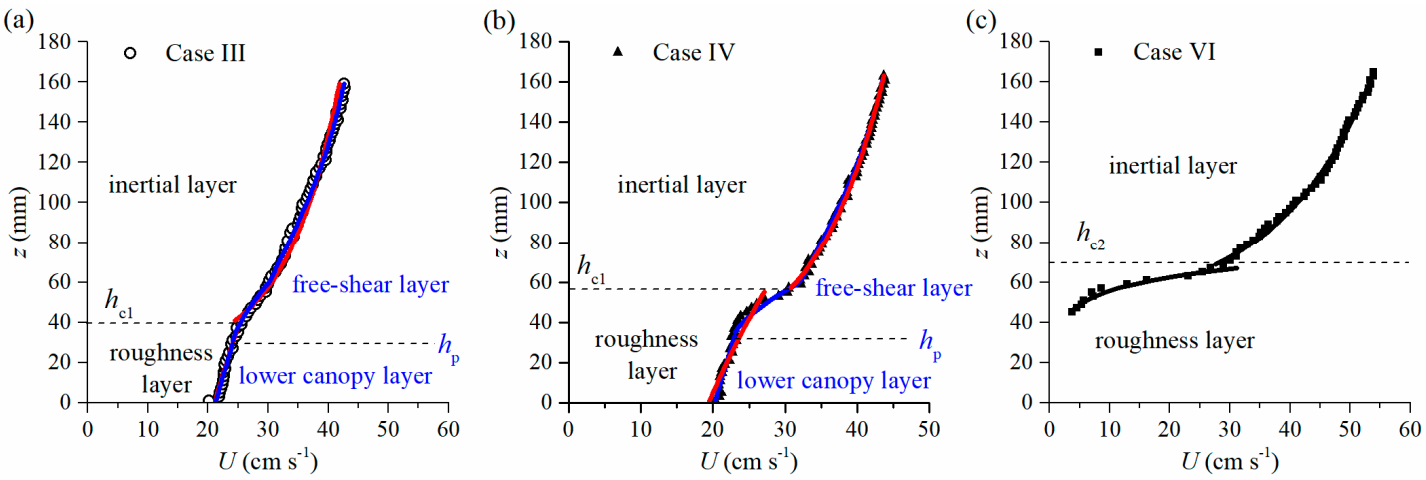

Figure 11. U Profiles along the depth for (a) Cases III, (b) Case IV and (c) Case VI.

As for the features of the bed-shear flow, the fitting results lead to $R^{2}=0.9811$ (Case III) and 0.997 (Case IV) (logarithmic law in the inertial layer) and 0.9247 (Case III) and 0.8213 (Case IV) (exponential law in the roughness layer) (see the red lines in Figure 11a,b). We find that the velocity dip layer disappears in this condition, since $U$ profile near the water surface could also be described approximately by the logarithmic law.

For flow in Case VI, experimental $U$ profile in the inertial layer $\left(h_{\mathrm{c} 2}<z<H\right.$ in Figure 11c) fits well with logarithmic function $\left(R^{2}=0.9947\right)$ (Figure 11c). The dense stems did not allow access of the ADV probes hence $U$ profile in the zone where $z<0.7 h_{\mathrm{v}}$ could not be presented in this case. Nezu \& Sanjou [9] have shown that $U$ is small there in this condition. Therefore, only the $U$ profile from $z=0.7 h_{\mathrm{v}}$ to $z=h_{\mathrm{c} 2}$ was considered. Results of the Least Square Fitting by the exponential function seems to be fine with $R^{2}=0.9641$.

\section{Discussion}

The vegetation density $\lambda$ controls turbulent flow type, which affects scalar dispersion and mass transport in the river. The main purpose of this study was to investigate flow at $0.04<\lambda<0.1$ and $\lambda>\lambda_{\mathrm{NB}}$, with the bed-shear flow and free-shear flow being laid out as the backdrop. Three-dimensional velocities were collected using ADV devices, and turbulent statistics (velocity, shear stress, skewness coefficients), turbulence kinetic energy generation rate, turbulence spectra and the velocity distribution laws were analyzed in the research.

\subsection{Horizontal Heterogeneity in the Flow Field}

The flow characteristics were studied basing on the overall flow dynamics that were represented by the horizontal-averaged velocities. Therefore, the heterogeneity of the flow field was described beforehand. The results show that the flow is mild behind vegetation, which is consistent with observations of the doubled-layered vegetation flow [22]. The flow resistance does not change above vegetation layer, so that the flow is uniform there in the horizontal plane. It is sufficiently accurate to describe the horizontally averaged velocity according to velocities at points $1 \#$ and 2\#, which supports the experimental methods conducted by previous researchers $[23,24]$. 


\subsection{Flow at $0.04<\lambda<0.1$}

For flow at $0.04<\lambda<0.1$, (1) the velocity increases considerably and the $G_{S}$ profile attains its vertical maximum both near the bed and at the canopy top; (2) the maximum $-\overline{u w}$ appears between the one for the bed-shear flow and the one for the free-shear flow; (3) $S k_{w}$ profile is similar to the one for the free-shear flow; however, $S k_{u}$ attains its vertical maximum at the bottom, which is in line with that for the bed-shear flow and (4) some slight humps are observed and the $F(f)$ tends to decrease with $f$ within the low-frequency range. Experimental $U$ data could be fitted to comply with either the free-shear flow feature or the bed-shear flow feature. Since $G_{S}$ at the bed surface is considerably smaller than the one near vegetation top, we conclude that the vegetation drag plays a dominant role to turbulent motion.

\subsection{The Secondary Boundary-Shear Flow}

For the secondary boundary-shear flow in Case VI, the densely arranged vegetation "raises" the equivalent bed to the vegetation top and causes the porous media flow within the canopy. Therefore, (1) the flow velocity is quite small within the canopy and increases sharply above canopy; (2) the $-\overline{u w}$ and $G_{S}$ are vertically maximum near canopy top; (3) the $S k_{u}$ and $S k_{w}$ profiles above canopy are similar to those throughout depth in the bed-shear flow, except that the sidewall effect disappears in the secondary boundary-shear flow; and (4) the spectral curve fluctuates intensely within the low-frequency range and spectral densities of low-frequency eddies are small within canopy.

The critical height where $U$ profile deviates from the logarithmic law (i.e., $z=h_{\mathrm{c} 2}$ ) nearly coincides with the point where bursting phenomenon changes, and the portion of the roughness layer above canopy occupies about $10 \%$ of the region from vegetation top to the free surface in Case VI (i.e., $h_{\mathrm{c} 2}-h_{\mathrm{v}} \approx 0.1\left(H-h_{\mathrm{v}}\right)$ ). These two points are similar to features of the bed-shear flow, since the vegetation top acts as a new boundary in this extremely dense vegetation condition. However, the secondary boundary-shear flow is significantly fiercer than the bed-shear flow, according to the profiles of turbulent statistics and $G_{S}$ in different cases.

\section{Conclusions}

The turbulent flow field has been explored experimentally with a range of submerged vegetation densities $(\lambda)$. Specific discussions about flow at $0.04<\lambda<0.1$ and $\lambda>\lambda_{\mathrm{NB}}$ have been conducted, according to vertical distributions of turbulent statistics (velocity, shear stress, skewness coefficients), turbulence kinetic generation rate and turbulence spectra. The findings are summarized as follows:

1. It is sufficiently accurate to represent overall flow characteristic by an average of velocities measured at Locations $1 \#$ \& 2\#;

2. For a modest value of $0.04<\lambda<0.1$, characteristics of profiles for turbulent statistics are similar to both the bed-shear flow one and the free-shear flow one. $U$ profile could be described to comply with either the free-shear flow or the bed-shear flow features;

3. The secondary boundary-shear flow occurs in Case VI with $\lambda=1.44$. U profile could be described to comply with the bed- shear flow feature, and the boundary between the roughness layer and the inertial layer locates above the canopy;

4. The change of turbulent flow type induced by an increase of $\lambda$ would intensify the turbulence with large maximum value of $G_{S}$. The point where turbulence is vertically fiercest moves upwards gradually with $\lambda$;

5. The ISRs of spectral curves range from 0.4 to $4 \mathrm{~Hz}$ at different heights except within canopy in the secondary boundary-shear flow. For spectral curves of the low-frequency eddies, there are some slight humps at $0.04<\lambda<0.1$, and the curves fluctuate intensely while $\lambda>\lambda_{\mathrm{NB}}$.

The accurate value of $\lambda_{\mathrm{NB}}$ should be determined by large series of experiments, with variations in vegetation configuration (stiffness, shape and arrangement pattern), flow velocity and submergence 
depth being considered. Turbulence structures in different conditions should also be studied basing on flow field measurement in the future.

Author Contributions: Conceptualization, H.Z. and J.Y.; methodology, H.Z., J.Y., J.L. and J.Z.; validation S.Y., J.L. and J.Z.; data curation, H.Z.; writing-original draft preparation, H.Z.; writing- review and editing, J.Y.; supervision, J.Y.

Funding: This research was funded by Fundamental Research Funds for the Central Universities [2017B618X14], the National Natural Science Foundation of China [51579079, 51979084, 51239003], the 111 Project [B17015], Ministry of Education and State Administration of Foreign Experts Affairs, P. R. China and Postgraduate Research \& Practice Innovation Program of Jiangsu Province [KYCX17_0425], China Scholarship Council project [No.201806715026].

Acknowledgments: Thanks to Bidya Sagar Pani for his help in considerably improving this article.

Conflicts of Interest: The authors declare no conflicts of interest.

\section{References}

1. Huai, W.X.; Li, C.G. Longitudinal dispersion in open channel flow with suspended canopies. Water Sci. Technol. 2016, 74, 722-728. [CrossRef] [PubMed]

2. Yang, Z.H.; Bai, F.P.; Huai, W.X.; An, R.D.; Wang, H.Y. Modelling open-channel flow with rigid vegetation based on two-dimensional shallow water equations using the lattice Boltzmann method. Ecol. Eng. 2017, 106, 75-81. [CrossRef]

3. Li, D.; Yang, Z.H.; Sun, Z.H.; Huai, W.X.; Liu, J.H. Theoretical model of suspended sediment concentration in a flow with submerged vegetation. Water 2018, 10, 1656. [CrossRef]

4. Yilmazer, D.; Ozan, A.Y;; Cihan, K. Flow characteristics in the wake region of a finite-length vegetation patch in a partly vegetated channel. Water 2018, 10, 459. [CrossRef]

5. Nepf, H.M. Flow and transport in regions with aquatic vegetation. Annu. Rev. Fluid Mech. 2012, 44, 123-142. [CrossRef]

6. Yan, J.; Dai, K.; Tang, H.W.; Cheng, N.S.; Chen, Y. Advances in research on turbulence structure in vegetated open channel flows. Adv. Water Sci. 2014, 18, 456-461.

7. Cheng, N.S.; Nguyen, H.T.; Tan, S.K.; Shao, S.D. Scaling of Velocity profiles for depth-limited open channel flows over simulated rigid vegetation. J. Hydraul. Eng. 2012, 138, 673-683. [CrossRef]

8. Wang, X.K.; Shao, X.J.; Li, D.X. Fundamental River Mechanics; Water \& Power Press: Beijing, China, 2002.

9. Nezu, I.; Sanjou, M. Turbulence structure and coherent motion in vegetated canopy open- channel flows. J. Hydro-Environ. Res. 2008, 2, 62-90. [CrossRef]

10. Caroppi, G.; Gualtieri, P.; Fontana, N.; Giugni, M. Vegetated channel flows: Turbulence anisotropy at flowrigid canopy interface. Geosciences 2018, 8, 259. [CrossRef]

11. Wang, H.; Tang, H.W.; Zhao, H.Q.; Zhao, X.Y.; Lv, S.Q. Incipient motion of sediment in presence of submerged flexible vegetation. Water Sci. Eng. 2015, 8, 63-67. [CrossRef]

12. Lv, S.Q.; Guo, M.J.; Yuan, L.W. Effects of submerged flexible vegetation on bed load movement in open channel. Yangtze River 2016, 47, 67-71.

13. Yan, J. Experimental Study on Flow Resistance and Turbulence Characteristics of Open Channel Flows with Vegetation. Ph.D. Thesis, Hohai University, Nanjing, China, 2008.

14. Nezu, I.; Nakagawa, H.; Tominaga, A. Secondary currents in a straight channel flow and the relation to its aspect ratio. Turbul. Shear Flows 1985, 4, 246-260.

15. Ghisalberti, M.; Nepf, H.M. Mixing layers and coherent structures in vegetated aquatic flows. J. Geophysical Res. 2002, 107. [CrossRef]

16. Belcher, S.; Jerram, N.; Hunt, J. Adjustment of a turbulent boundary layer to a canopy of roughness elements. J. Fluid Mech. 2003, 488, 369-398. [CrossRef]

17. Katul, G.G.; Poggi, D.; Ridolfi, L. A flow resistance model for assessing the impact of vegetation on flood routing mechanics. Water Resour. Res. 2011, 47, W08533. [CrossRef]

18. Ei-Hakim, O.; Salama, M.M. Velocity distribution inside and above branched flexible roughness. J. Irrig. Drain. Eng. 1992, 118, 914-927. [CrossRef]

19. Wilson, J.D. A second-order closure model for flow through vegetation. Bound. Layer Meteorol. 1988, 42, 371-392. [CrossRef] 
20. Nikora, V.; Koll, K.; Mclean, S.; Dittrich, A.; Aberle, J. Zero-plane displacement for rough-bed open-channel flows. In Proceedings of the River Flow 2002, Louvain-la-Neuve, Belgium, 4-6 September 2002.

21. Baptist, M.J.; Babovic, V.; Uthurburu, J.R.; Keijzer, M.; Uittenbogaard, R.E.; Mynett, A.; Verwey, A. On inducing equations for vegetation resistance. J. Hydraul. Res. 2007, 45, 435-450. [CrossRef]

22. Liu, D.; Diplas, P.; Hodges, C.C.; Fairbanks, J.D. Hydrodynamics of flow through double layer rigid vegetation. Geomorphology 2010, 116, 286-296. [CrossRef]

23. Huai, W.X.; Zeng, Y.H.; Xu, Z.G.; Yang, Z.H. Three-layer model for vertical velocity distribution in open channel flow with submerged rigid vegetation. Adv. Water Resour. 2009, 32, 487-492. [CrossRef]

24. Tang, H.W.; Tian, Z.J.; Yan, J.; Yuan, S.Y. Determining drag coefficients and their application in modelling of turbulent flow with submerged vegetation. Adv. Water Resour. 2014, 69, 134-145. [CrossRef]

(C) 2019 by the authors. Licensee MDPI, Basel, Switzerland. This article is an open access article distributed under the terms and conditions of the Creative Commons Attribution (CC BY) license (http://creativecommons.org/licenses/by/4.0/). 